\title{
INVERSIÓN UTERINA PUERPERAL CRÓNICA: PRESENTACIÓN DE CASO Y REVISIÓN DE LA LITERATURA
}

\author{
Chronic uterine inversion: a case \\ report and review of the literature \\ Jorge A. Rodríguez, M.D.*, Adriana P. Calderón, M.D.**, \\ Luis Alfonso López, M.D.***, Daniel Montenegro, M.D.**** \\ Recibido: mayo 11/05 - Revisado: junio 30/05 - Aceptado: agosto 25/05
}

\section{RESUMEN}

Se presenta un caso de diagnóstico tardío de inversión uterina poco frecuente en la literatura médica. El cuadro clínico de la paciente incluía sangrado genital moderado persistente y dispareunia de 14 meses de evolución. La técnica de reposicionamiento uterino se realizó por vía vaginal con revisión posterior por vía laparoscópica y evolución satisfactoria. Se hace una revisión de la literatura.

Palabras clave: inversión uterina, complicación postparto.

\section{SUMMARY}

A rare case of late diagnosis of uterine inversion is presented. Clinically, the patient had profuse vaginal bleeding and dyspareunia of 14 months duration. The replacement of the uterus was done by vaginal technique and revised by laparoscopic technique with satisfactory evolution. A review of the literature is presented.

Key words: uterine inversion, postpartum complication.

\footnotetext{
* Ginecoobstetra. Hospital Simón Bolívar. Clínica Palermo. Clínica El Bosque. Docente Universidad El Bosque, Correo electrónico: jarobg@yahoo.com.

** Residente Ginecoobstetricia IV año. Universidad El Bosque.

*** Ginecoobstetra. Jefe Unidad Endoscopia Ginecológica. Hospital Simón Bolívar. Docente Universidad El Bosque.

**** Ginecoobstetra. Director Académico Programa Ginecoobstetricia. Universidad El Bosque. Hospital Simón Bolívar.
}

\section{CASO CLÍNICO}

Paciente de 20 años que acudió al servicio de consulta externa del Hospital Simón Bolívar, de la ciudad de Bogotá, por cuadro clínico de 14 meses de sangrado genital persistente posterior a parto vaginal.

La revisión de la historia clínica no mostró antecedentes personales ni familiares de importancia; perfil obstétrico G2P2A0V2C0. Su último parto fue atendido en casa por "partera", con referencia de posible inversión uterina durante el alumbramiento como complicación, que al parecer no requirió un manejo inmediato de urgencia y con el paso del tiempo se hizo crónica-persistente, debido a que la paciente no consultó a personal especializado. La paciente fue valorada y hospitalizada desde el servicio de urgencias para programación quirúrgica. Se reservaron glóbulos rojos empaquetados, crioprecipitados y plasma fresco congelado.

$\mathrm{Al}$ examen bajo anestesia se encontró una masa que protruía hasta el orificio vaginal, no friable, dura, de $9 \mathrm{~cm}$, con escaso sangrado genital no fétido; se palpaba un anillo fibrótico entre el orificio cervical y el cuerpo uterino; las paredes vaginales estaban libres y los anexos no eran palpables. Inmediatamente diferentes especialistas realizaron pruebas manuales múltiples para reposicionar el útero por la vía vaginal, con resultados fallidos (gráfica 1). Se realiza entonces una histerotomía longitudinal sobre 
la cara anterior e inferior del útero, incluyendo el anillo fibroso de constricción con resección de un segmento de $0,5 \times$ x 0,5 cm y colocación de puntos de reparo. Posteriormente se realiza la eversión del órgano (gráficas 2 a 6).

La evolución posquirúrgica fue satisfactoria, la paciente requirió transfusión de 2 unidades de glóbulos rojos por hallazgos de hematocrito y hemoglobina en límite inferior en las pruebas de análisis clínico de ingreso; sin embargo el sangrado intraoperatorio fue escaso (aproximadamente $150 \mathrm{cc}$ ).

El seguimiento posoperatorio de la paciente a la segunda y cuarta semanas fue satisfactorio, revelando un examen físico sin alteraciones con un útero en AVF y anexos de características normales. Actualmente la paciente se encuentra asintomática. El reporte de biopsia realizado intraoperatoriamente reveló tejido fibrótico benigno sin características infecciosas.

\section{INTRODUCCIÓN}

La inversión del útero es una entidad clínica rara que se presenta como una emergencia obstétrica y constituye un desafío diagnóstico en la ginecología. La asociación entre patología maligna (carcinoma endometrial, sarcoma) e inversión uterina es aún más rara. El diagnóstico exacto antes del tratamiento es esencial para ofrecer el manejo apropiado.

El conocimiento de la anatomía uterina se remonta al año 2500 a.C.; las escrituras en papiros de la época indican que los antiguos egipcios consideraban una distinción entre el útero y la vagina. ${ }^{1}$ Antes de la momificación a los cadáveres que tenían prolapso genital se les debía reposicionar el útero a su lugar apropiado, ya que la disección era evitada en ese período de tiempo; así fue como se dieron cuenta de que el útero y la vagina eran dos órganos separados. ${ }^{2}$

La condición de inversión uterina fue antiguamente confundida con el prolapso y es a Hipócrates (460 - 370 a.C.) a quien se ha dado el crédito de ser el primero en reconocerla. Fue Sorano (200 d.C.) quien no sólo definió claramente la inversión, sino que concluyó que podía ser ocasionada por la

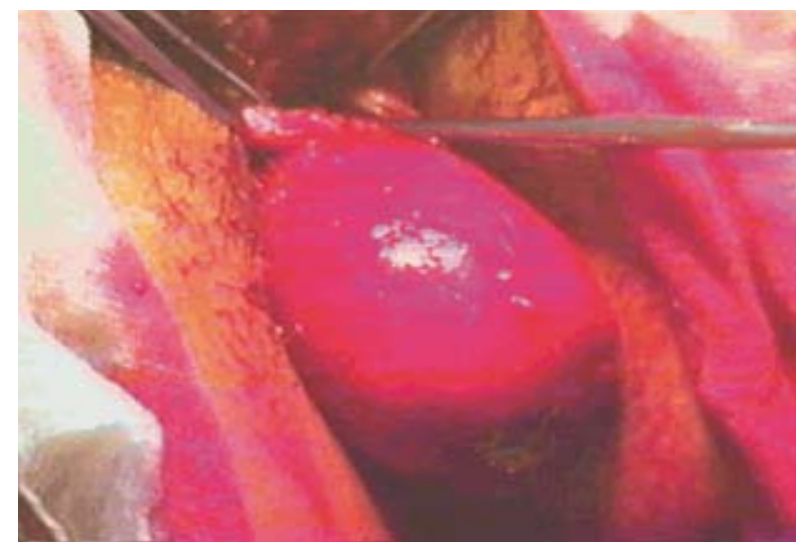

Gráfica 1. Visualización de hallazgos descritos al examen inicial bajo anestesia.

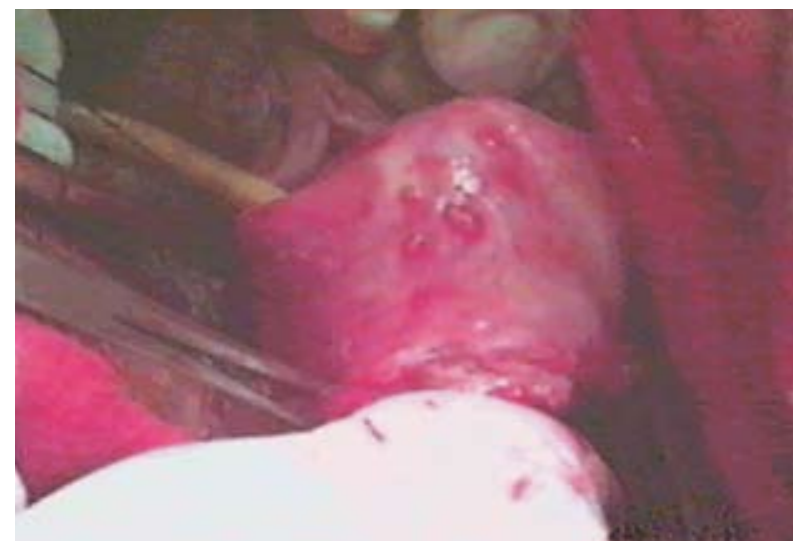

Gráfica 2. Histerotomía longitudinal sobre la cara anterior e inferior del útero, incluyendo el anillo fibroso de constricción con resección de un segmento de $0,5 \times 0,5 \mathrm{~cm}$ y colocación de puntos de reparo. Posteriormente se realiza la eversión del órgano.

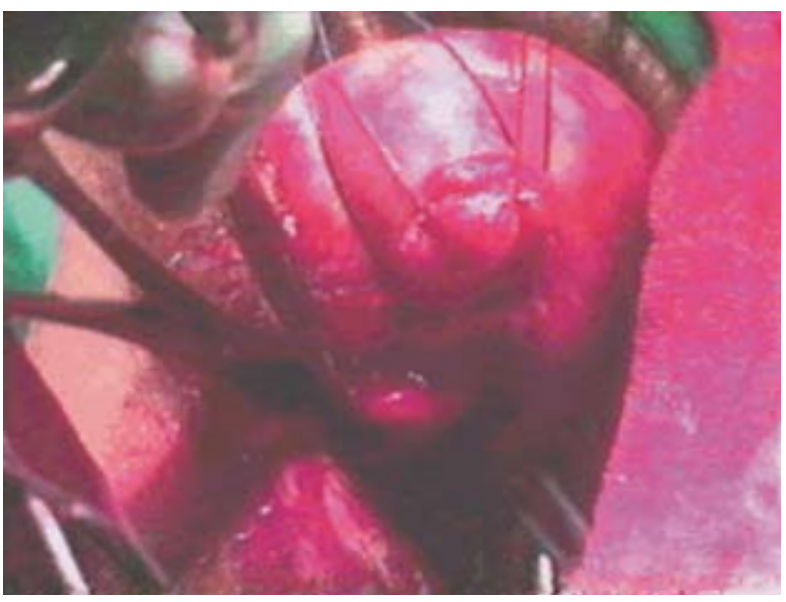

Gráfica 3. Cierre de la histerotomía por planos con cat-gut cromado 1 hasta el anillo fibroso de constricción. 


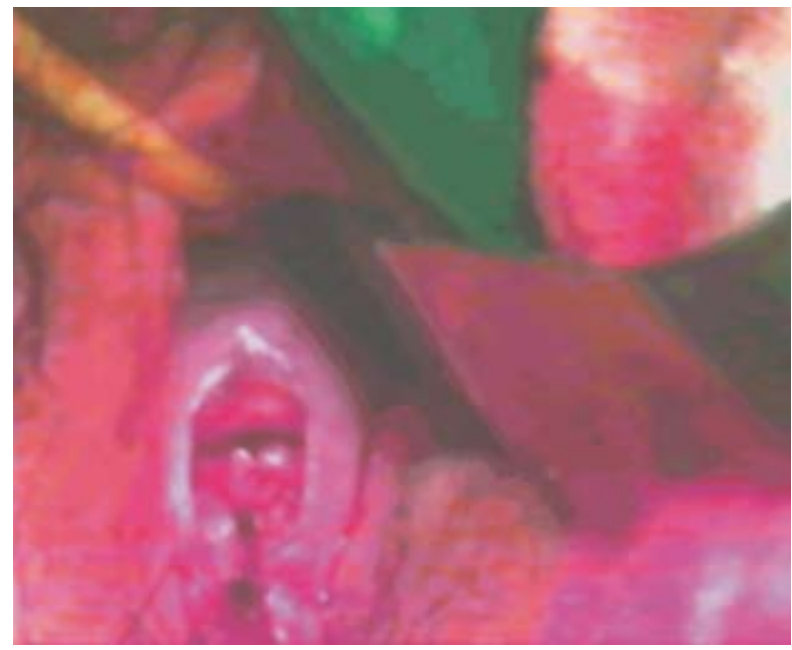

Gráfica 4. Apertura del espacio vésico-uterino y ubicación del útero en la cavidad pélvica.

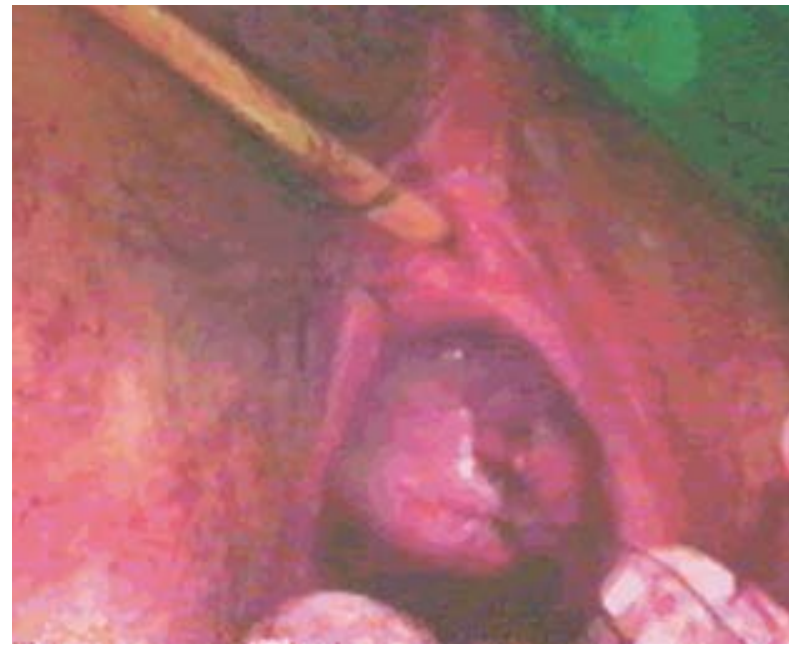

Gráfica 5. Terminación de la histerorrafia hasta el cuello.

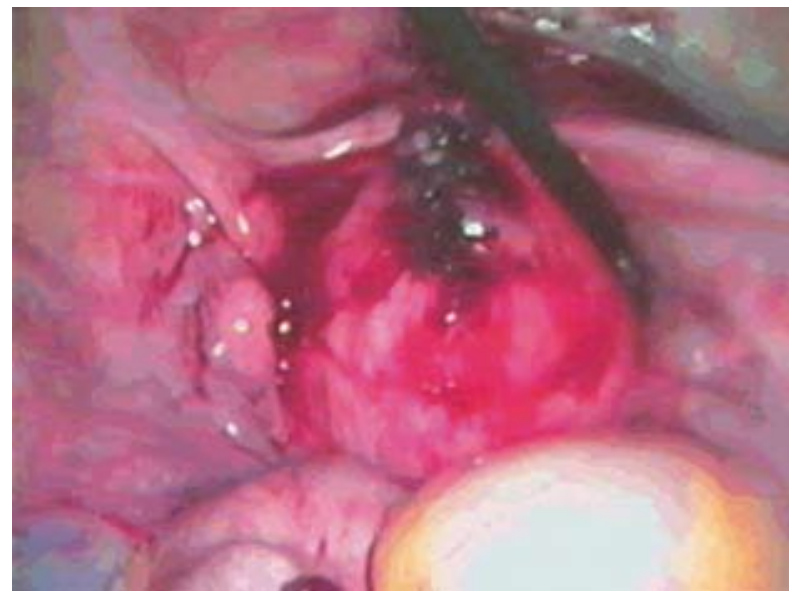

Gráfica 6. Revisión por vía laparoscópica del útero (hemostasia y posición). tracción del cordón placentario. Posteriormente Avicenna en Arabia (980 - 1037 d.C.) dio una clara descripción del diagnóstico diferencial entre inversión y prolapso. La próxima referencia importante de inversión uterina ocurrió durante el tiempo de Ambroise Pare (siglo XVI) en el que se le reconoció como una entidad diferente al prolapso genital. ${ }^{3}$

\section{METODOLOGÍA}

La búsqueda de la literatura se realizó por medio de Pubmed, sin ningún tipo de límite; usando el término "inversión uterina" se obtuvieron 242 referencias, y con el término "inversión uterina crónica", 19 citas bibliográficas. Debido a la baja prevalencia de esta condición, la literatura disponible no es extensa. Por otro lado, se encuentran artículos publicados desde hace aproximadamente 40 - 50 años, de difícil consecución y los más recientes se encuentran publicados en revistas que no son de fácil obtención.

\section{EPIDEMIOLOGÍA}

La inversión uterina puerperal es una complicación excesivamente rara y muy seria que ocurre en el tercer estadio del trabajo de parto; los grandes estudios reportan una incidencia de 1:20.000 - 1:25.000 partos (la estadística más frecuente indica 1:1.860 después de cesárea a 1:3.737 partos vaginales). ${ }^{3-5}$

La inversión uterina no puerperal es aún más rara, y en la literatura los datos estadísticos de incidencia varían según la serie de casos. ${ }^{6}$ En la serie de casos de McCullagh el 85\% fue de origen puerperal y el $15 \%$ fue no puerperal. En la variedad puerperal el 8,6\% fue de origen súbito. ${ }^{7}$

Estos datos estadísticos también varían dependiendo del área geográfica. En India se reporta una incidencia de 1:8.537 partos, en Estados Unidos 1:23.127 y en los hospitales británicos de $1: 27.902 .^{8}$

\section{CLASIFICACIÓN}

La inversión uterina se clasifica considerando varios aspectos, entre los cuales figura su relación con el parto, el tiempo y la extensión de la inversión. 
Con relación al parto se puede clasificar en puerperal y no puerperal, siendo esta última casi siempre crónica. ${ }^{7}$ La mayoría de casos reportados asociados a inversiones crónicas se deben a tumores benignos o malignos del cuerpo uterino, comúnmente miomas submucosos $(71,6 \%)$, pólipos endometriales, sarcomas uterinos y cervicales $(13,6 \%)$, cáncer endometrial $(6,8 \%)$ y pacientes ancianas con antecedente de amputaciones altas del cuello uterino. ${ }^{9}$ La inversión uterina es considerada idiopática (8\%) cuando no se encuentra ninguna causa. ${ }^{10}$

Según la duración en tiempo desde el parto hasta el momento del diagnóstico se considera que la inversión es aguda en las primeras 24 horas puerperales, subaguda de 24 horas hasta el primer mes y crónica por más de 4 semanas. ${ }^{11}$

La clasificación más popular está basada en la extensión de la inversión (tabla 1). ${ }^{11,12}$

\begin{tabular}{|c|l|}
\hline Tabla 1. Clasificación de la inversión uterina \\
según la extensión
\end{tabular}

El reporte más temprano de inversión uterina no puerperal fue reportado por Simpson en 1887, en un caso asociado con malignidad uterina (sarcoma). ${ }^{13}$ En la inversión uterina puerperal, el cuello uterino debe estar dilatado y el músculo liso relajado para permitir la protrusión del útero, por ende ocurre más frecuentemente en el tercer estadio del trabajo de parto. ${ }^{14}$ Los factores involucrados en la etiología son la tracción del cordón umbilical con una placenta que no se ha separado, presión fúndica inapropiada y remoción manual de la placenta. ${ }^{15}$ Las condiciones obstétricas que pueden predisponer a esta patología incluyen un cordón umbilical corto, placenta fúndica, anomalías uterinas y placenta muy adherida a la pared.

Van Vugt y cols. describieron factores predisponentes en la inversión uterina puerperal que incluyen la calidad de la pared uterina, ligamentos y pared abdominal, inserción de la placenta en el fondo uterino, baja paridad y edad joven. También describieron algunos factores provocadores tales como la alta presión intrabdominal, la tracción del cordón umbilical y las fuerzas externas que actúan directamente en el útero. ${ }^{11}$

Las principales características de la inversión uterina aguda son hemorragia abundante, choque (35\%) y dolor. $\mathrm{Al}$ examen físico, dependiendo del grado de inversión del útero, se puede evidenciar una masa roja que protruye o no por el orificio vaginal, y a la palpación abdominal no se encuentra el fondo uterino. ${ }^{16}$

El síntoma más frecuente de inversión uterina crónica es la hemorragia irregular y flujo vaginal. La paciente suele referir "la presencia de un tumor en la vagina" o "algo" protruyendo por ésta. El diagnóstico es difícil incluso con el examen físico.

El útero completamente invertido forma un bulto piriforme que ocupa la parte superior del canal vaginal, es liso, color rojo oscuro y usualmente propenso a sangrar con la palpación; los orificios de las trompas de Falopio rara vez pueden distinguirse. Se palpa un anillo de constricción alrededor del cuello de la protuberancia que representa el orificio externo, y revela que el canal cervical no está completamente invertido. Si el cuello uterino está completamente invertido, no se palpa anillo de constricción y el dedo pasa sobre la superficie lisa del fondo de saco vaginal.

La palpación bimanual revela la ausencia del cuerpo uterino en su posición normal. El método rectoabdominal es el más recomendado ya que la 
vagina está ocupada por el útero invertido. En la palpación bajo anestesia, de los bordes de la inversión pueden ser reconocidos y son útiles para la reducción de la misma. ${ }^{17}$

También han sido utilizados como métodos diagnósticos la ecografía pélvica, TAC y resonancia magnética. ${ }^{18-20}$

Las complicaciones más frecuentes son los émbolos pulmonares (debido a la estasis vascular), la infección y la anemia crónica. ${ }^{21}$

\section{Diagnóstico diferencial}

El diagnóstico prequirúrgico de la inversión uterina crónica es difícil y generalmente se realiza intraoperatoriamente. Se deben tener en cuenta los siguientes diagnósticos diferenciales: ${ }^{10}$

- Prolapso de mioma uterino submucoso

- Tumor mulleriano o prolapso de sarcoma uterino pediculado

- Cáncer de cuello uterino

- Prolapso útero vaginal

\section{TRATAMIENTO}

El tratamiento de la inversión puerperal aguda debe ser manejado agresivamente con líquidos y sangre si está disponible (tabla 2). ${ }^{12,22}$

\section{Tabla 2. Manejo de la inversión} puerperal aguda

Soporte:

Líquidos agresivos, dos líneas venosas de acceso, reemplazo sanguíneo, sonda de Folley, tratamiento del dolor, manejo multidisciplinario (anestesia, enfermería, etc.).

\section{Reposición del útero:}

Anestesia general, tocolíticos para relajar el útero ( $\mathrm{MgSO}_{4}$ o terbutalina), antibióticos profilácticos.

\section{Técnicas:}

No quirúrgicas (Johnson, O’Sullivan), quirúrgicas (Huntington y Haultain (abdominales), Spinelli (vaginal).

\section{Posterior a reposición de útero:}

Masaje uterino, oxitocina, prostaglandinas (F2a o misoprostol).
El tratamiento apropiado de la inversión uterina crónica depende del diagnóstico prequirúrgico, siendo la histerectomía con o sin salpingooforectomía bilateral el recomendado para pacientes con paridad satisfecha. ${ }^{23,24}$

O' Sullivan describió una técnica no quirúrgica para la reposición del útero invertido que consiste en conectar a la vagina una ventosa que genera una presión hidrostática graduada y progresivamente va reduciendo el útero invertido. ${ }^{25}$

El procedimiento de Huntington es una técnica quirúrgica realizada por laparotomía, en la cual se identifica el fondo uterino invertido, se repara con pinzas de Allis y se aplica una tracción ascendente hasta que se reposicione el útero. ${ }^{12,22}$

La técnica más popular y recomendada para pacientes con deseo de paridad es el procedimiento de Haultain, el cual se realiza si hay fracaso en la técnica de Huntington; consiste en incidir el anillo cervical por vía vaginal y posteriormente se reposiciona el útero por tracción fúndica (vía abdominal). ${ }^{24}$ Los estudios muestran que después de la corrección quirúrgica por este método el embarazo y el parto son posibles. ${ }^{26}$

La técnica de Spinelli se realiza por vía vaginal mediante una incisión mediana en la parte invertida del útero hasta reducir el anillo de constricción. ${ }^{27}$

Cuando se asocia un estado de malignidad, la cirugía de elección depende de la estadificación de la enfermedad. La histerectomía abdominal con biopsias adecuadas usualmente está indicada. Algunos autores recomiendan la escisión de la masa tumoral antes de la histerectomía. El pronóstico depende del diagnóstico inicial y del estado de la enfermedad. ${ }^{28}$

\section{DISCUSIÓN}

En la literatura no se encuentra ningún caso reportado de inversión uterina puerperal crónica, ya que la mayoría ocurren de forma aguda como complicación del parto amenazando la vida de la paciente. La inversión uterina crónica se ha 
relacionado con causas no puerperales, es decir, con patologías ginecológicas (miomas, pólipos, carcinoma endometrial, sarcoma, etc.), pero su incidencia no se ha estimado en la literatura ya que su presentación es muy rara y depende de la serie de casos.

La etiología de la inversión uterina crónica es idiopática o relacionada con un proceso tumoral (benigno o maligno), cuyo mecanismo fisiopatológico consiste en una dilatación de la cavidad uterina, debilitamiento de las paredes y contracciones expulsivas del útero provocadas por el propio peso del tumor, produciendo sangrado irregular y descarga vaginal crónica. En casos de presentación idiopática las manifestaciones clínicas aparecen en forma abrupta, dada por dolor y choque, ${ }^{30}$ síntomas que no presentaba la paciente en mención. En nuestro caso, las manifestaciones clínicas se asemejan a la forma crónica pero su etiología fue puerperal, sin ningún evento ginecológico previo, por lo que hubiéramos esperado que las manifestaciones ocurrieran de forma aguda. Recomendamos la inspección vaginal de rutina en todos los partos para hacer un diagnóstico de la inversión uterina en forma temprana, y poder así tratar inmediatamente el shock y reposicionar el útero invertido en esta dramática situación para generar una mínima morbilidad materna.

La inversión uterina crónica, a pesar de su rara presentación, se debe tener en cuenta como diagnóstico diferencial en el marco de un contexto clínico apropiado, y el diagnóstico prequirúrgico permite planear un adecuado tratamiento.

Los métodos para reposicionar el útero invertido se basan en técnicas quirúrgicas y no quirúrgicas, las cuales se deben seleccionar de forma individualizada, dependiendo del tipo de inversión uterina y paridad de la paciente.

\section{REFERENCIAS}

1. Medvei VC. A history of clinical endocrinology. New York: The Parthenon Publishing Group; 1993.

2. Speroff L, Glass RH, Kase NG. Clinical gynecologic endocrinology and infertility. Baltimore: Lippincott Williams \& Wilkins; 1999.

3. Das P. Inversion of the uterus. J Obstet Gynaecol Br Emp 1940;47:525-48.

4. Fahmy K. Acute inversion of the uterus. Int Surg 1977;62:100-2.

5. Baskett TF. Acute uterine inversion: a review. J Obstet Gynaecol Can 2002;24:953-6.

6. Moodley M, Moodley J. Non-puerperal uterine inversion in association with uterine sarcoma: clinical management. Int Gynecol Cancer 2003;13:244-5.

7. McCullagh WMH. Inversion of the uterus: a report of three cases and an analysis of 233 recently recorded cases. J Obstet Gynaecol Br Emp 1925;32:280-97.

8. Mehra U, Ostapowicz F. Acute puerperal inversion of the uterus primipara. Obstet Gynecol 1976;47:30S-32S.

9. Mwinyoglee J, Simelela N, Marivate M. Nonpuerperal uterine inversions. A two case report and review of the literature. Cent Afr J Med 1997;43:268-71.

10. Gowri V. Uterine inversion and corpus malignancies: a historical review. Obstet Gynecol Surv 2000;5 5:703-7.

11. Van Vugt PJ, Baudoin P, Blom VM, van Deursen CT. Inversio uteri puerperalis. Acta Obstet Gynaecol Scand 1981;60:353-62.

12. Thomson AJ, Greer IA. Non-haemorrhagic obstetric shock. Baillieres Best Pract Res Clin Obstet Gynaecol 2000;14:19-41.

13. Simpson. Cyclopaedia of Obstetrics and Gyanecology. New York: William Wood \& Co, 1887, ix, pp 335.

14. Bell JE Jr, Wilson GF, Wilson LA. Puerperal inversion of the uterus. Am J Obstet Gynecol 1953;66:767-80.

15. Irani S, Jordan J. Management of uterine inversion. Curr Obstet Gynaecology 1997;7:232-5.

16. Kochenour NK. Intrapartum obstetric emergencies. Crit Care Clin 1991;7:851-64.

17. Krenning RA, Dorr PJ, de Groot WH, de Goey WB. Non-puerperal uterine inversion. Case report. Br J Obstet Gynaecol 1982;89: 247-9.

18. Gross RC, McGahan JP. Sonographic detection of partial uterine inversion. AJR Am J Roentgenol 1985;144:761-2.

19. Lewin JS, Bryan PJ. MR imaging of uterine inversion. J Comput Assist Tomogr 1989;13:357-9. 
20. Salomon CG, Patel SK. Computed tomography of chronic non puerperal uterine inversion. J Comput Assist Tomogr 1990;14:1024-6.

21. Schulman JM, Stanton JS. Acute nonpuerperal uterine inversion. South Med J 1981;74:1142-5.

22. Calder AA. Emergencies in operative obstetrics. Baillieres Best Pract Res Clin Obstet Gynaecol 2000; 14:43-55.

23. Rocconi R, Huh WK, Chiang S. Postmenopausal uterine inversion associated with endometrial polyps. Obstet Gynecol 2003;102:521-3.

24. Lascaraides E, Cohen M. Surgical management of non-puerperal inversion of the uterus. Obstet Gynecol 1968;32:376-81.
25. Tan KH, Luddin NS. Hydrostatic reduction of acute uterine inversion. Int J Gynecol Obstet 2005;91:63-4.

26. Sinha G, Sinhan A. Fertility and reproduction following inversion of the uterus. J Indian Med Assoc 1993; 91:149-50

27. Milenkovick M, Kahn J. Inversion of the uterus: a serious complication at childbirth. Acta Obstet Gynecol Scand 2005;84:94-99.

28. Kioses E, Pilalis A, Hatzioannou L, Moulopoulos G, Rodolakis A. Acute complete nonpuerperal uterine inversion. Acta Obstet Gynecol Scand 2004;83:870.

Conflicto de intereses: ninguno declarado. 